\title{
ChemComm
}

\section{Spin transport properties of triarylamine-based nanowires}

Cite this: Chem. Commun., 2014 50,6626

Received 6th March 2014

Accepted 5th May 2014

DOI: $10.1039 / c 4 c c 01710 b$

www.rsc.org/chemcomm

\author{
Sandip Bhattacharya, ${ }^{*}$ Akinlolu Akande and Stefano Sanvito
}

Triarylamine-derivatives can self-assemble upon light irradiation in onedimensional nanowires with remarkable hole transport properties. We use a combination of density functional theory and Monte Carlo simulations to predict the nanowires spin-diffusion length. The orbital nature of the nanowires valence band, namely a singlet $\pi$-like band localised on $\mathrm{N}$, suggests that hyperfine coupling may be weak and that spin-orbit interaction is the primary source of intrinsic spin relaxation. Thus, we construct a model where the spin-orbit interaction mixes the spins of the valence band with that of three degenerate lower valence bands of $\mathrm{sp}^{2}$ nature. The model includes also electron-phonon interaction with a single longitudinal mode. We find a room temperature spin-diffusion length of the order of $100 \mathrm{~nm}$, which increases to $300 \mathrm{~nm}$ at $200 \mathrm{~K}$. Our results indicate that triarylamine-based nanowires are attractive organic semiconductors for spintronics applications.

Functionalized triarylamines are a family of molecules well known in organic electronic applications such as light-emitting diodes, field-effect transistors and more recently multilayered heterojunctions solar cells. ${ }^{1,2}$ Triarylamine derivatives dispersed in chloroform solution, when exposed to light, self-assemble in one dimensional (1D) supramolecular nanowire structures. ${ }^{3}$ Even more interesting is the fact that the nanowires can be grown across nanogaps so that two-terminal self-assembled devices can be made. Intriguingly such devices display low resistances, with ohmic-like current-voltage, $I-V$, characteristics. This points to the nanowires being good charge conductors, ${ }^{4}$ i.e. being potentially useful for electronic applications.

In this communication we report on a first principle calculation of the spin-diffusion length of one of such nanowires, i.e. we provide an assessment on whether or not triarylamines derivatives can be used as materials platform for organic spintronics. ${ }^{5}$ In particular, we focus on nanowires forming from the precursor depicted in Fig. 1(a), for which hole transport experimental data are available, ${ }^{4}$ and for which we have recently estimated ${ }^{6}$ room temperature hole mobility of the order of $10 \mathrm{~cm}^{2} \mathrm{~V}^{-1} \mathrm{~s}^{-1}$, for a pristine defect free nanowire structure.

School of Physics, AMBER and CRANN Institute, Trinity College, Dublin 2, Ireland. E-mail: sbhattac@tcd.ie

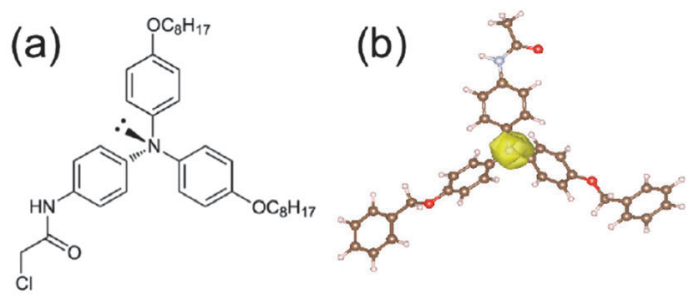

Fig. 1 The triarylamine derivative used to construct the nanowires investigated in this work. In panel (a) we show a diagram of the molecule and in (b) we present an isosurface plot of the charge density associated to the HOMO of the molecule in the gas phase. Note the $p_{z}$ character of such molecular orbital.

The electronic structure of the molecule in the gas phase is calculated by using density functional theory (DFT), at the level of the hybrid B3LYP functional, ${ }^{7}$ for the molecule's geometry optimized by conjugate gradient (to forces smaller than $2 \times 10^{-5}$ Hartree per Bohr). In particular we use the DFT numerical implementation of the Gaussian09 suit $^{8}$ and the $6-31 G^{*}$ basis set. We obtain a gap between the highest occupied molecular orbital (HOMO) and the lowest unoccupied molecular orbital (LUMO) of $4.1 \mathrm{eV}$. Most importantly for the discussion here the HOMO is an orbital singlet strongly localized on the central $\mathrm{N}$ atom of the triarylamine unit. An orbital decomposition reveals its strong $\mathrm{p}_{z}$ character, as demonstrated by the charge density isosurface of Fig. 1(b). Note that the orbital character of the HOMO does not change even for the +1 radical. Furthermore, for both the neutral and the +1 charging state the remaining part of the HOMO manifold is at least $1 \mathrm{eV}$ below such $\mathrm{p}_{z}$ orbital.

Next we move to calculating the nanowire electronic structure. This is constructed by placing the optimized molecule in an orthorhombic cell with a co-facial arrangement. The cell $c$ lattice parameter coincides with the intermolecular distance and $a=b \gg c$ in order to avoid spurious interactions between periodic images. The geometry is further optimized with the Grimme's exchange correlation functional, ${ }^{9}$ which includes semi-empirical dispersive forces into the standard generalized gradient approximation (GGA). Calculations are performed with the Quantum Espresso package, ${ }^{10}$ using Vanderbilt ultra-soft pseudopotentials, ${ }^{11}$ a plane wave 


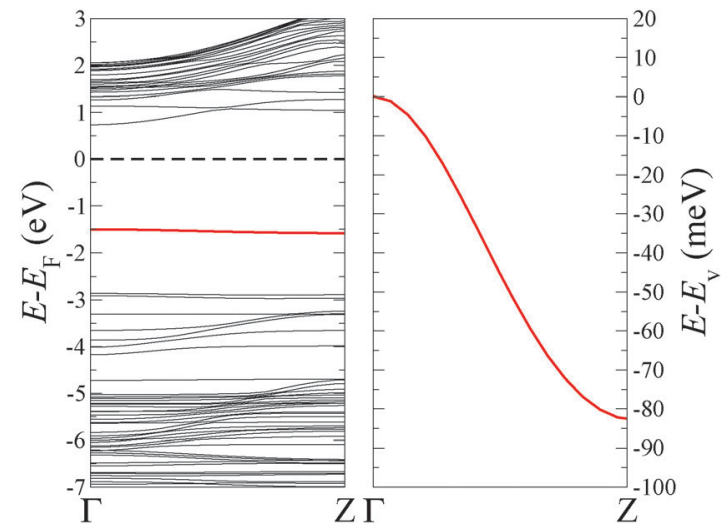

Fig. 2 Band structure of the triarylamine derivative-based single nanowire (left-hand side panel). The band plotted in red at about $1.5 \mathrm{eV}$ from the Fermi level, $E_{\mathrm{F}}$, (set to $0 \mathrm{eV}$ ) is derived from the $\mathrm{HOMO}$ of the molecule in the gas phase depicted in Fig. 1(b). The right-hand side panel is a zoom of such $\mathrm{HOMO}$ band, which reveals the almost perfect cosine-like curvature. In this case the energy zero is set at the top of the valence band, $E_{\mathrm{v}}$.

cut-off 30 Ry and a $5 \times 5 \times 5$ Monkhorst-Pack grid with a 0.01 Ry Gaussian broadening.

The bandstructure of the nanowire is presented in Fig. 2. The most notable feature is the presence of a well-separated valence band (in red) with a distinctive cosine-like dependence [see the right-hand side panel of Fig. 2]. This lies about $1.5 \mathrm{eV}$ above the rest of the valence manifold and approximately $2.3 \mathrm{eV}$ below the lowest of the conduction bands. The local density of states associated to such band returns us an orbital, which essentially coincides with that shown in Fig. 1(b) for the molecule in the gas phase. This means that the valence band is derived entirely from the molecular HOMO and that the leading interaction is $\operatorname{pp} \sigma$ ( $\sigma$ bonds formed by $\mathrm{p}_{z}$ orbitals) in nature. The bandwidth is narrow, $\sim 80 \mathrm{meV}$, as a consequence of the rather large intermolecular separation $c=4.8 \AA$.

Note that the bandgap calculated with the Grimme's functional is significantly smaller than the HOMO-LUMO gap calculated with B3LYP for the gas phase. This is due to the well-known selfinteraction problem, which is partially corrected by B3LYP. However, the important features here are the valence band curvature and its bandwidth. These are both little affected by self-interaction and in fact the Grimme's and B3LYP results are rather similar. ${ }^{6}$ We now proceed to constructing an effective nearest-neighbour tight-binding Hamiltonian based on the calculated DFT electronic structure, which will then be used to evaluate the spin-transport properties.

We consider a minimal $\mathrm{s}-\mathrm{p}$ model of four degrees of freedom per molecule. These comprise of the $\mathrm{p}_{z}$ orbital, defining the valence band, and three degenerate and occupied $\mathrm{sp}^{2}$-like orbitals. Note that $\mathrm{p}_{z}$ is responsible for the hole conductivity, while the $\mathrm{sp}^{2}$ contribute to the spin-mixing. The Hamiltonian of the problem can be written as

$$
\begin{aligned}
\hat{H}= & \sum_{i I \neq j J, \sigma}\left[t_{i I j J}+\alpha_{i I j J}\left(q_{I}-q_{J}\right)\right]\left(c_{i I \sigma}^{\dagger} c_{j J \sigma}+\text { h.c. }\right) \\
& +\sum_{i I, \sigma} \varepsilon_{i}\left(c_{i I \sigma}^{\dagger} c_{i I \sigma}+\text { h.c. }\right)+\hat{H}_{\mathrm{SO}}+\sum_{I J} \frac{1}{2} K\left(q_{I}-q_{J}\right)^{2},
\end{aligned}
$$

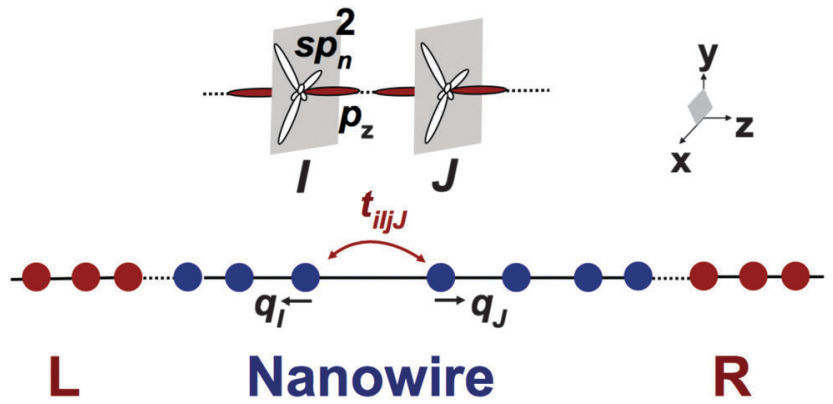

Fig. 3 Tight-binding model used to calculate the nanowires spindiffusion length. This comprises four orbitals per site (per molecule), respectively three planar $\mathrm{sp}^{2}$ and one perpendicular $\mathrm{p}_{z}$. The lower panel describes the setup used for the transport calculations, where a nanowire is sandwiched between two semi-infinite $1 D$ leads, respectively $L$ and $R$. The molecules in the nanowire are represented as blue circles. The electrodes inject uncorrelated spin-polarized carriers into the $p_{z}$ bands $(\mathrm{HOMO})$ of the nanowire. Note that the $z$-axis is the transport direction.

where $c_{i I \sigma}^{\dagger}\left(c_{i I \sigma}\right)$ creates (annihilates) an electron at the $I$-th molecule in the orbital $i$ with spin $\sigma=\uparrow, \downarrow$. A schematic illustration of our model is presented in Fig. 3.

Due to the crystal field the on-site energies are identical for the $\mathrm{sp}^{2}$ orbitals, $\varepsilon_{\mathrm{sp}^{2}}=-10.3 \mathrm{eV}$, but different from that of the $\mathrm{p}_{z}, \varepsilon_{\mathrm{p}_{z}}=$ $-8.0 \mathrm{eV}$. These are evaluated from the on-site energies of the atomic orbitals of $\mathrm{N}$ at the GGA level. We will see later that the amount of spin mixing is determined by $\varepsilon_{\mathrm{sp}^{2}}-\varepsilon_{\mathrm{p}_{z}}$ and not by their absolute values. The hopping integral between $\mathrm{p}_{z}$ orbitals is $1 / 4$ of the valence band bandwidth (1D system), $t_{\mathrm{p}_{z} I \mathrm{p}_{z} J}=21 \mathrm{meV}$. The other nearest neighbour hopping integrals have the following amplitudes, $t_{\mathrm{sp}^{2} I \mathrm{sp}^{2} J}$ $=-0.4 \mathrm{meV}$ and $t_{\mathrm{p}_{z} I \mathrm{sp}^{2} J}=2 \mathrm{meV}$. These have been computed by standard tight binding construction from their constituent atomic orbitals, ${ }^{13}$ which, in turn, have been obtained from the GGA bandstructure of a $1 \mathrm{D} \mathrm{N}$ chain with the same $\mathrm{N}-\mathrm{N}$ distance as that between the central $\mathrm{N}$ atoms of neighbouring molecules in the relaxed nanowire geometry. Note that since the $\mathrm{N}$ atoms are all co-axial the $\mathrm{p}_{z}-\mathrm{sp}^{2}$ and $\mathrm{sp}^{2}-\mathrm{sp}^{2}$ hopping parameters are much smaller than the $\mathrm{p}_{z}-\mathrm{p}_{z}$ one.

The Hamiltonian comprises of three other terms. The first one is an additional contribution to the hopping parameter, $\alpha_{i j j}\left(q_{I}-q_{J}\right)$. This is proportional to the atomic displacement of the molecules about their equilibrium positions, $\left\{q_{I}\right\}$ and describes the electronic coupling to crystal vibrations. ${ }^{12}$ Here we consider $\alpha_{i j j} \neq 0$ and identical for all orbitals, noting that the $\mathrm{sp}^{2}$ bands do not contribute to the transport, so that the precise value of $\alpha_{i j J}$ is irrelevant for such manifold. Such electron-phonon energy is counterbalanced by a restoring classical vibration energy [last term in eqn (1)], with elastic constant $K$. Estimates for $\alpha$ and $K$ are from our previous work. ${ }^{6}$ Here we consider only longitudinal Peierls-like phonon modes, i.e. oscillation in the relative position of the molecules along the nanowire axis ( $z$ in Fig. 3). Finally, $\hat{H}_{\text {SO }}$ is the spin-orbit (SO) coupling, which takes the form

$$
\hat{H}_{\mathrm{SO}}=\lambda_{\mathrm{SO}} \sum_{i j \alpha \beta I} c_{i I \alpha}^{\dagger} \hat{\overrightarrow{\vec{L}}}_{I}^{i j} \cdot \vec{\sigma}^{\alpha \beta} c_{j I \beta},
$$

where $\hat{\overrightarrow{L_{I}}}$ is the angular momentum operator for the $I$-th molecule, $\vec{\sigma}$ is a vector of Pauli matrices and $\lambda_{\text {so }}$ is the SO 
strength. This latter quantity is extracted from the SO split of the $\mathrm{p}$ orbitals of a $\mathrm{N}$ atom in the gas phase as calculated from a DFT atomic simulation (at the GGA level). Thus, it is set to be $\lambda_{\text {so }}=5 \times 10^{-3} \mathrm{eV}$. Since the SO potential is very short range, we consider only on-site contributions to the SO matrix. These can be explicitly written on our $\mathrm{p}_{z}-\mathrm{sp}^{2}$ basis by decomposing the $\vec{L} \cdot \vec{\sigma}$ operator into its constituent angular momenta and spin ladder operators.

$\hat{H}_{\text {SO }}$ creates an on-site (in each given molecule) potential mixing the different spin components. Such mixing is achieved by allowing orbital transitions that preserve the total angular momentum but do not conserve its third component. As such, spin is conserved in the electron hopping between different molecules, so that the influence of the temperature on the spin mixing manifests itself through the temperature dependence of the mobility. An additional possible source of intrinsic spin relaxation is hyperfine interaction. However, we believe that this is weak for the nanowires under investigation. In fact, the valence band is entirely determined by a $\mathrm{N}-\mathrm{p}_{z}$ orbital, meaning that the relevant hyperfine-active ion is $\mathrm{N}$. Nitrogen has a nuclear magnetic moment of $\mu_{\mathrm{N}}=0.14 \mu_{\mathrm{H}}$, i.e. it is only $14 \%$ of the nuclear moment of $\mathrm{H}$. As such here we do not consider the effect of hyperfine coupling.

Note that, in principle, even in absence of SO interaction spin diffusion can occur due to extrinsic sources like paramagnetic impurities and defects. However, here we consider perfect single-crystalline structures, i.e. we describe only the intrinsic spin-scattering properties of the nanowires. This is a well-justified approximation since the nanowires are free of transition metals and so is their synthesis.

The quantity that we wish to estimate is the spin-diffusion length, $l_{\mathrm{s}}$, i.e. the average distance travelled by a spin before changing its orientation. This can be extracted from a two-terminal transport calculation, whose setup is schematically presented in Fig. 3. Two semi-infinite leads inject uncorrelated spin-polarized electrons into the nanowire valence (HOMO) band. The electrodes are onedimensional atomic chains described by a nearest-neighbour tightbinding Hamiltonian with one orbital per site and hopping parameter $\gamma$. The condition $|\gamma| \gg\left|t_{i j j J}\right|$ establishes the wideband limit, in which the density of state of the electrode is approximately constant on the energy scale of the nanowire valence band. The two-probe conductance of such device is then extracted by using the standard non-equilibrium Green's function formalism, following the numerical prescription of ref. 14. In particular here we integrate the energy-dependent conductance, $G(E)$, over the entire valence band.

The two-probe conductance can be decomposed into four components

$$
G=G_{\mathrm{sc}}+G_{\mathrm{sm}} \text { with } G_{\mathrm{sc}}=\sum_{\sigma} G_{\sigma \sigma} ; G_{\mathrm{sm}}=\sum_{\sigma} G_{\sigma-\sigma} .
$$

Here $G_{\sigma, \sigma^{\prime}}$ represents the conductance due to carriers entering the nanowire with spin $\sigma$ and being transmitted with spin $\sigma^{\prime}$, so that $G_{\text {sc }}$ is the spin-conserving part of the conductance, while $G_{\mathrm{sm}}$ is the spin-mixing one. In absence of SO interaction $G_{\mathrm{sm}}=0$, but when $\lambda_{\text {so }} \neq 0$ spin mixing is expected. In our setup one of the electrodes inject electrons with only one spin (ideal half-metal) and here we calculate the fraction of electrons, which have changed such spin direction while traveling through the nanowire. The relevant quantity is the spin polarization (SP) of the current, which can be written as

$$
\mathrm{SP}=\frac{I_{\uparrow}-I_{\downarrow}}{I_{\uparrow}+I_{\downarrow}}=\frac{G_{\mathrm{sc}}-G_{\mathrm{sm}}}{G_{\mathrm{sc}}+G_{\mathrm{sm}}},
$$

where $I_{\sigma}$ is the current carried by the electrons with spin $\sigma$. The equality then follows from linear response, $I=G V$, with $V$ being the voltage. The spin-diffusion length is extracted from the dependence of SP on the nanowire length, $L$, namely $\mathrm{SP}=\mathrm{SP}_{0} \mathrm{e}^{-L / l_{\mathrm{s}}}$, with $\mathrm{SP}_{0}$ being the spin polarization of the injected electrons. Such definition is consistent with the standard practice adopted in spin valves experiments of fitting the reduction of the magnetoresistance with length to an exponential decay. ${ }^{5}$ Since the magnetoresistance is directly proportional to SP and since SP is directly available to us, here we use the spin polarization to extract $l_{\mathrm{s}}$. Finally, in order to estimate $l_{\mathrm{s}}$ at finite temperature, the classical lattice displacements, $\left\{q_{i}\right\}$ are evolved via Monte Carlo simulations ${ }^{15}$ and the spin transport observables are averaged over 20000 Monte Carlo steps.

Fig. 4(a) shows the first principles estimate of SP as a function of $L$ for three different temperatures, $193 \mathrm{~K}, 290 \mathrm{~K}$ and $387 \mathrm{~K}$. Clearly, as a consequence of the spin-mixing, SP is reduced as the length of the wire increases. The reduction is much more evident at high temperature, when scattering is more pronounced and so is spin-scattering. A fit of SP to an exponential function provides the spin-diffusion length, which is plotted as a function of temperature in Fig. 4(b). Here one finds that $l_{\mathrm{s}}$ drops from $332 \mathrm{~nm}$ at $193 \mathrm{~K}$ to $22 \mathrm{~nm}$ at well above room temperature $(387 \mathrm{~K})$, i.e. it drops by about one order of magnitude in this temperature range. The room temperature value for $l_{\mathrm{s}}$ is approximately $100 \mathrm{~nm}$, which is rather large for one-dimensional organic materials. ${ }^{16}$ Interestingly, in the same temperature range the nanowires mobility reduces from $\sim 19 \mathrm{~cm}^{2} \mathrm{~V}^{-1} \mathrm{~s}^{-1}$ (193 K) to $\sim 8 \mathrm{~cm}^{2} \mathrm{~V}^{-1} \mathrm{~s}^{-1}(387 \mathrm{~K}),{ }^{6}$ indicating that the spin-diffusion

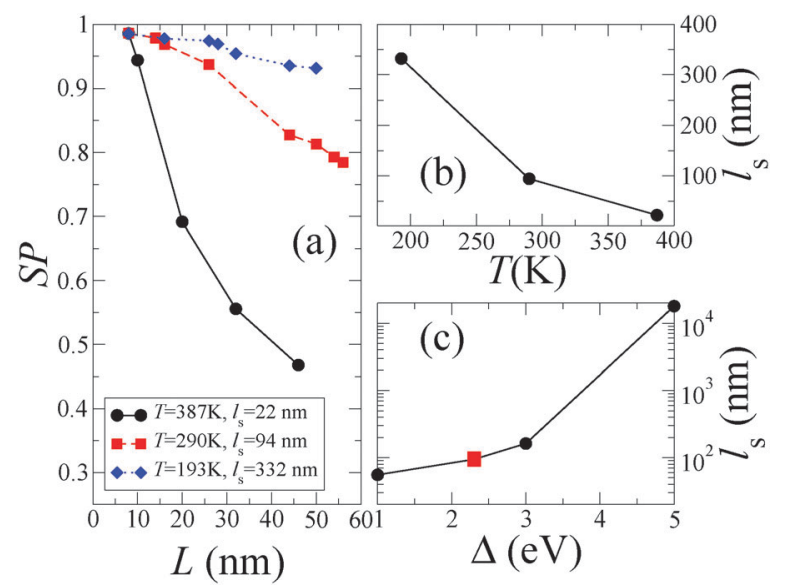

Fig. 4 Calculated spin-transport properties of the triarylamine-based nanowires. In panel (a) we show SP as a function of the nanowire length, $L$, for three different temperatures. The corresponding fits for $l_{s}$ are reported. Panel (b) shows the temperature dependence of $l_{s}$, while in (c) we present $l_{s}$ against the difference between the on-site energies of the $\mathrm{sp}^{2}$ and $\mathrm{p}_{z}$ orbitals, $\Delta . \ln (\mathrm{c})$ the red square is for the DFT-calculated $\Delta$. 
length in this case is rather sensitive to the mobility, as expected from SO-driven spin-mixing. ${ }^{5}$

Finally, we discuss the dependence of our results on the electronic structure parameters of the model. As mentioned before the SO coupling allows orbital transitions, which conserve the total angular momentum but not its third component. For our $\mathrm{p}_{z}-\mathrm{sp}^{2}$ Hamiltonian this means that the effectiveness of the SO interaction to mix spins depends on the hybridization between the $\mathrm{p}_{z}$ and the $\mathrm{sp}^{2}$ bands. This is controlled by the ratio between the relevant hopping parameter and the difference in on-site energy between the $\mathrm{p}_{z}$ and the $\mathrm{sp}^{2}$ bands, $\Delta=\varepsilon_{\mathrm{p}_{z}}-\varepsilon_{\mathrm{sp}^{2}}$. When extracting the various parameters from DFT, the hopping integral $t_{\mathrm{p}_{z} I \mathrm{p}_{z} J}$ is well determined by the valence band bandwidth, while the others can be calculated easily from a linear chain of $\mathrm{N}$ atoms with lattice separation corresponding to that of the $\mathrm{N}$ atoms in the nanowires. The on-site energies, however, depend on the crystal field and need to be evaluated from the electronic structure of the nanowire alone. This is simple for the $\mathrm{p}_{z}$ band, but significantly more complicated for the $\mathrm{sp}^{2}$ ones, since they are positioned in a spectral region populated by many other closely spaced bands [see Fig. 2].

The value $\Delta=2.3 \mathrm{eV}$ used so far has been extracted by looking at the density of state for the nanowire projected over the planar $\mathrm{N}-\mathrm{p}$ orbital $\left(\mathrm{p}_{x}\right.$ and $\mathrm{p}_{y}$ ), while Fig. 4(c) displays $l_{\mathrm{s}}$ at $290 \mathrm{~K}$ as a function of $\Delta$. As expected $l_{\mathrm{s}}$ increases as $\Delta$ gets larger, i.e. as the hybridization between bands formed by $\mathrm{p}$ orbitals with different orientation is reduced. Such change in $l_{\mathrm{s}}$ is quite drastic and for $\Delta=5 \mathrm{eV}$ one calculates a room temperature spin-diffusion length of the order of $10 \mu \mathrm{m}$. In contrast when $\Delta$ is halved with respect to the $a b$ initio estimate, $l_{\mathrm{s}}$ is reduced to approximately $50 \mathrm{~nm}$. Since the separation between the on-site energies can be tuned by the strength of the ligand field acting on the planar $\mathrm{sp}^{2}$ orbitals, the results presented in Fig. 4(c) suggest a chemical strategy for increasing the spin-diffusion length in such nanowires, namely that of coordinating the central $\mathrm{N}$ atom by molecules producing a strong ligand field in the plane orthogonal to the nanowire axis.

In conclusion we have constructed a $\mathrm{p}_{z}-\mathrm{Sp}^{2}$ model describing the electronic structure of triarylamine-based nanowires and studied their intrinsic spin-transport properties. When the parameters of the model are extracted from accurate DFT calculations we find a spin-diffusion length of the order of $100 \mathrm{~nm}$ at room temperature, with the spin scattering being due to SO interaction. These are rather large values when compared to other organic materials. Furthermore, we have shown that one can enhance such spin-diffusion length by tuning the electronic properties of the nanowire. For instance our calculations show that a potential strategy is that of designing ligands that promote a strong ligand field in the plane perpendicular to the wire axis.

The authors are grateful to Graeme Watson for the Gaussian09 package. This work is supported by Science Foundation of Ireland under the grant 07/IN.1/I945, the CSET grant underpinning CRANN and by the European Research Council under EU's 7th Framework Programme (FP/2007-2013)/ERC Grant Agreement no. 307891. Computational resources have been provided by TCHPC and ICHEC.

\section{References}

1 S. Kim, J. K. Lee, S. O. Kang, J. Ko, J. H. Yum, S. Fantacci, F. De Angelis, D. Di Censo, M. K. Nazeeruddin and M. Grätzel, J. Am. Chem. Soc., 2006, 128, 16701-16707.

2 Z. Ning, Q. Zhang, W. Wu, H. Pei, B. Liu and H. Tian, J. Org. Chem., 2008, 73, 3791-3797.

3 E. Moulin, F. Niess, M. Maaloum, E. Buhler, I. Nyrkova and N. Giuseppone, Angew. Chem., Int. Ed., 2010, 49, 6974-6978.

4 V. Faramarzi, F. Niess, E. Moulin, M. Maaloum, J.-F. Dayen, J.-B. Beaufrand, S. Zanettini, B. Doudin and N. Giuseppone, Nat. Chem., 2012, 4, 485-490.

5 S. Sanvito, Chem. Soc. Rev., 2011, 40, 3336-3355.

6 A. Akande, S. Bhattacharya, T. Cathcart and S. Sanvito, J. Chem. Phys., 2014, 140, 074301.

7 C. Lee, W. Yang and R. G. Parr, Phys. Rev. B, 1988, 37, 785-789; A. D. Becke, J. Chem. Phys., 1993, 98, 5648-5652.

8 M. J. Frisch, et al., Gaussian 09, Revision A.01, Gaussian Inc, Wallingford CT, 2009.

9 S. Grimme, J. Comput. Chem., 2006, 27, 1787-1799.

10 P. Giannozzi, et al., J. Phys.: Condens. Matter, 2009, 21, 395502.

11 D. Vanderbilt, Phys. Rev. B, 1990, 41, 7892-7895.

12 W. P. Su, J. R. Schrieffer and A. J. Heeger, Phys. Rev. Lett., 1979, 42, 1698-1701; W. P. Su, J. R. Schrieffer and A. J. Heeger, Phys. Rev. B, 1980, 22, 2099-2111.

13 A. Sutton, Electronic Structure of Materials, Oxford University Press, 1993, pp. 117-119.

14 I. Rungger and S. Sanvito, Phys. Rev. B, 2008, 78, 035407.

15 S. Bhattacharya, M. S. Ferreira and S. Sanvito, J. Phys.: Condens. Matter, 2011, 23, 316001.

16 V. A. Dediu, L. E. Hueso, I. Bergenti and C. Taliani, Nat. Mater., 2009, 8, 707-716. 